\title{
Glossário de Termos Usados em Química Medicinali,ii
}

Emília Sousa*

Madalena Pinto

Jorge Salvador

M. Matilde Marques

Rui Moreira

\begin{abstract}
Glossary of Terms Used in Medicinal Chemistry. The terminology Medicinal Chemistry has been the target of several translations into Portuguese, as reflected in the designation of the courses that minister its contents in Portugal. The existence of a glossary in Portuguese resulting from the translation and adaptation of the glossaries of terms used in Medicinal Chemistry in accordance with IUPAC recommendations of 1998 and 2013 is crucial to harmonize emerging concepts in this field. The need for these glossaries and their updating was felt by IUPAC groups due to rapid changes in Medicinal Chemistry because of advances in genomics and molecular biology and also of the need to establish international standards of definitions. The purpose of translating these glossaries is to provide in a single document a terminology in Portuguese idiom consistent with concise definitions of terms covering the various aspects of Medicinal Chemistry.
\end{abstract}

\begin{abstract}
A terminologia Medicinal Chemistry tem sido alvo de várias traduções para o português, como refletido nas diferentes designações das disciplinas que ministram o seu conteúdo em Instituições Portuguesas. A existência de um glossário em português, resultante da tradução e adaptação dos glossários de termos utilizados em Química Medicinal de acordo com as recomendações da IUPAC de 1998 e 2013 é importante para harmonizar conceitos emergentes neste campo. A necessidade dos glossários referidos e da sua atualização foi sentida pelos grupos IUPAC devido às rápidas mudanças na Química Medicinal como consequência dos avanços na genómica e biologia molecular e também pela necessidade de estabelecer padrões internacionais de definições. O objetivo de traduzir estes glossários é fornecer num documento único uma terminologia em português, consistente com definições concisas de termos abrangendo os vários aspetos da Química Medicinal.
\end{abstract}

\begin{abstract}
Introdução
A Química Medicinal, do inglês Medicinal Chemistry é definida de acordo com a IUPAC (International Union of Pure and Applied Chemistry) [1] como uma: "Disciplina baseada na química, envolvendo também aspetos da biologia, medicina, e ciências farmacêuticas. Engloba a invenção, descoberta, conceção, identificação e preparação de compostos biologicamente ativos, o estudo do seu metabolismo, a interpretação do seu modo de ação a nível molecular e o estabelecimento de relações estrutura-atividade."

A terminologia Medicinal Chemistry tem sido alvo de diversas traduções para português, como é o reflexo da designação das unidades curriculares que ministram os seus conteúdos, sendo as mais frequentes Química Medicinal, Química Farmacêutica e Química Terapêutica. O termo, Química Medicinal, já expressa que a disciplina compreende três aspetos importantes: i) a conceção de novas moléculas (aspeto medicinal), ii) a síntese
\end{abstract}

de novas substâncias (aspeto químico sintético) e iii) o estudo farmacêutico, farmacodinâmico e farmacocinético do composto biologicamente ativo e/ou potencial fármaco (aspeto farmacêutico).

Neste artigo é incluído um glossário que constitui parte integrante do "Glossário de Termos Usados em Química Medicinal" de acordo com as recomendações da IUPAC, publicadas nos gilossários de 1998 [1] e 2013 [2]. 0 objetivo da tradução conjunta destes glossários é fornecer num documento único a terminologia em português consistente com definições concisas de termos, abrangendo os vários aspetos da Química Medicinal e que são utilizados ao longo do documento. 0 texto apresentado pretende mostrar o início e o fim do glossário, contendo as entradas correspondentes às letras A e B e as últimas correspondentes às letras V e X. As restantes entradas podem ser consultadas como material suplementar no g'lossário de termos completo, disponível online em spq.pt/files/files/BSPQ/N158-artigo-22.pdf. 


\section{Ordem alfabética de entrada}

\section{1. Ácido nucleico [1]}

Macromolécula composta por sequências lineares de nucleótidos que desempenham várias funções nas células vivas, ex., armazenamento de informação genética e sua transferência de uma geração para a seguinte (ADN; ácido desoxirribonucleico), expressão desta informação na síntese proteica (ARNm, ARNt) e podem atuar como componentes funcionais de unidades subcelulares, como ribossomas (ARNr).

O ARN (ácido ribonucleico) contém D-ribose e o ADN contém 2-desoxi-D-ribose como açúcar constituinte.

\section{Address-message concept [1] Ver mensagem-endereço.}

\section{ADME [1]}

Acrónimo de absorção, distribuição, metabolismo, excreção.

Ver também disponibilidade de fármacos, farmacocinética.

\section{ADMET [2]}

Acrónimo referente ao perfil ou processos de absorção, distribuição, metabolismo, excreção e toxicidade para um xenobiótico após a sua administração in vivo.

Nota: ADME também é usado para delinear esses parâmetros selecionados no contexto do perfil farmacocinético de um xenobiótico. Na medida em que qualquer uma das cinco características se pode tornar um obstáculo durante o desenvolvimento de fármacos, o perfil de ADMET é geralmente estudado e otimizado entre análogos eficazes numa etapa inicial do processo de descoberta de fármacos usando modelos in vitro para prever tais comportamentos em estudos clínicos.

Ver também farmacocinética, distribuição de fármacos.

\section{Análise comparativa de campo molecular (comparative molecular field analysis, CoMFA) [1]}

Método de 3D-QSAR que usa técnicas de correlação estatística para a análise da relação quantitativa entre a atividade biológica de um conjunto de compostos com um determinado alinhamento e as suas propriedades eletrónicas e estereoquímicas tridimensionais. Outras propriedades, como a hidrofobia e ligacções de hidrogénio, podem também ser incorporadas na análise.

Ver também relações quantitativas entre estrutura tridimensional e atividade (3D-QSAR).

\section{Afinidade [1]}

Tendência de uma molécula se associar a outra. A afinidade de um fármaco corresponde à sua capacidade para se ligar a um alvo biológico (recetor, enzima, sistema transportador, etc.). Para recetores farmacológicos pode ser vista como a frequência com que um fármaco, quando se aproxima de um recetor por difusão, permanecerá numa posição de energia livre mínima no campo de força desse recetor.

Para um agonista (ou para um antagonista), a representação numérica da afinidade é o inverso da constante de equilíbrio de dissociação do complexo ligando-recetor denotada como $K_{A}$ e calculada como o quociente da constante de velocidade de dissociação $\left(k_{-}\right)$ dividida pela constante de velocidade de associação $\left(k_{1}\right)$.

\section{Agente biológico [2]}

Produto farmacêutico baseado num biopolímero, como uma proteína, aplicável à prevenção, tratamento ou cura de doenças ou lesões em humanos.

Nota: os agentes biológicos podem ser quaisquer vírus, soro terapêutico, toxina, antitoxina, vacina, componente ou derivado do sangue, produto alergénico ou produtos análogos.

\section{Agente carcinogénico [2]}

Agente (químico, físico ou biológico) que é capaz de aumentar a incidência de neoplasmas malignos, causando cancro.

\section{Agonista [1]}

Substância endógena ou um fármaco que pode interatuar com um recetor e iniciar uma resposta fisiológica ou farmacológica característica desse recetor (contração, relaxamento, secreção, ativação enzimática, etc.).

\section{Agonista inverso [1]}

Fármaco que atua no mesmo recetor de um agonista, mas produz um efeito oposto. Também designado como antagonista negativo.

\section{Agonista parcial [1]}

Agonista que é incapaz de induzir a ativação máxima de uma população de recetores, independentemente da quantidade de fármaco aplicado.

Ver também atividade intrínseca.

\section{Alerta estrutural [2]}

Características químicas presentes num composto hit ou composto-líder, indicativas de potencial toxicidade.

Nota: tipicamente, tais características incluem funcionalidades quimicamente reativas e componentes conhecidos por serem metabolizados a entidades quimicamente reativas. Exemplos incluem anidridos, aminas aromáticas e epóxidos. 
13. Alvo [2]

Ver alvo molecular.

\section{Alvo molecular [2]}

Proteína (ex. recetor, enzima, ou canal iónico), ARN ou ADN que está implicado num distúrbio clínico ou na propagação de qualquer evento indesejável.

Nota: geralmente estão disponíveis informações bioquímicas, farmacológicas ou genómicas que suportam o papel de tal alvo na doença.

15. Alvo terapêutico (druggable target) [2] Ver capacidade para alvo terapêutico, druggability.

\section{Análise de Hansch [1] \\ Investigaação da relação quantitativa entre a atividade biológica de uma série de compostos e os parâmetros físico-químicos dos seus substituintes ou globais que representam efeitos hidrofóbicos, eletrónicos, estereoquímicos e outros, usando a metodologia de regressão e correlação múltipla.}

\section{Análogo $[1,2]$}

Composto químico que possui semelhança estrutural com um composto de referência.

Nota: apesar da semelhança estrutural, um análogo pode apresentar propriedades químicas e/ ou biológicas diferentes, como é muitas vezes intencionalmente o caso, durante a conceção e a síntese, para otimizar quer a eficácia quer as propriedades ADMET de uma dada série [2].

Ver também descoberta de fármacos baseada em análogos, congénere, fármaco seguinte (follow-on drug).

\section{Análogo do estado de transição [1]}

Composto que mimetiza o estado de transição da reação entre um substrato e uma enzima.

\section{Analogue-based drug discovery [2]} Ver descoberta de fármacos baseada em análogos.

\section{Antagonista [1]}

Fármaco ou composto que apresenta efeitos fisiológicos opostos aos de outro. A nível do recetor, é uma entidade química que se opõe às respostas associadas ao recetor, normalmente induzidas por outro agente bioativo.

\section{Antagonista alostérico [2]}

Composto que se liga a um recetor num local separado, mas acoplado ativamente ao do agonista endógeno, para reduzir ativamente os sinais do recetor.

Nota: Os termos "antagonista alostérico" e "antagonista não competitivo" são frequentemente usados como sinónimos, mas não é necessariamente o caso.

Ver também antagonista não competitivo.

22. Antagonista neutro (em farmacologia) [2] Ligando que bloqueia as respostas de um recetor a agonistas e agonistas inversos com a mesma intensidade. Liga-se ao recetor sem provocar qualquer alteração de conformação ou mudança na razão das conformações ativadas para inativadas.

Nota: 0 antagonismo neutro ideal é difícil de atingir.

\section{Antagonista não competitivo [2]}

Antagonista funcional que se liga irreversivelmente a um recetor ou a um local distinto do local do agonista natural.

Ver antagonista alostérico.

\section{Antimetabolito [1]}

Análogo estrutural de um intermediário (substrato ou coenzima) de uma via metabólica fisiológica que atua substituindo o substrato natural e, deste modo, bloqueia ou desvia a biossíntese de substâncias fisiologicamente importantes.

25. Área de superfície polar (polar surface area, PSA), área de superfície polar topológica [2]

Área da superfície de uma molécula abrangendo todos os átomos polares (geralmente oxigénio e nitrogénio) e incluindo quaisquer átomos de hidrogénio que lhes estejam ligados.

Nota: a área de superfície polar é um parâmetro frequentemente utilizado (cf. descritor molecular) para a otimização da permeabilidade celular. Moléculas com uma PSA superior a 1,4 nm² são geralmente ineficientes a permear membranas celulares. Para que as moléculas penetrem a barreira hematoencefálica, $a$ área de superfície polar deve ser normalmente menor do que $0,6 \mathrm{~nm}^{2}$, embora possam ser tolerados valores até $0,9 \mathrm{~nm}^{2}$.

Ver também barreira hematoencefálica.

26. ARNi [2]

Ver Interferência por ARN (ARNi, ARN interference, RNAi).

27. ARNsi [2]

Ver pequeno ARN interferente (small inhibitory double-stranded RNA, small interfering double-stranded RNA, siRNA).

28. Árvore de Topliss [1]

Esquema operacional para o desenho de análogos. 


\section{Atividade constitutiva [2]}

Efeito de um recetor ou enzima exibido na ausência de um agonista ou ativador, respetivamente.

\section{Atividade intrínseca [1]}

Resposta estimuladora máxima induzida por um composto em relação à de um composto de referência.

Ver também agonista parcial.

Este termo evoluiu com o uso generalizado. Foi introduzido por Ariëns como um fator de proporcionalidade entre a resposta do tecido-alvo e a ocupação do recetor. 0 valor numérico da atividade intrínseca (alfa) pode variar entre a unidade (para agonistas totais, isto é, agonistas induzindo a resposta máxima do tecido-alvo) e zero (para antagonistas); os valores dentro desse intervalo evidenciam agonistas parciais. Na definição original de Ariëns, a natureza molecular de alfa só equivale à resposta máxima quando a resposta é uma função linear de ocupação do recetor. Esta função tem sido verificada. Assim, a atividade intrínseca, que é um parâmetro do fármaco e do tecido-alvo, não pode ser usada como um parâmetro característico do fármaco para a classificação de fármacos ou recetores de fármacos. Para esse fim deverá ser utilizada a eficácia relativa, ou seja, um fator de proporcionalidade derivado de métodos nulos. Finalmente, a "atividade intrínseca" não deve ser usada em vez de "eficácia intrínseca". Um "agonista parcial" deve ser denominado de "agonista com eficácia intrínseca intermédia" num determinado tecido-alvo.

\section{Atropoisómero [2]}

Estereoisómero resultante da rotação impedida em torno de uma ligacção simples em que o impedimento estereoquímico a essa rotação é suficiente para permitir o isolamento de isómeros individuais.

\section{Autacoide [1]}

Substância biológica segregada por várias células cuja atividade fisiológica está localizada na vizinhança do seu local de libertação; muitas vezes é referido como hormona local.

\section{Autoindução [2]}

Capacidade de um fármaco para induzir enzimas que medeiam o seu próprio metabolismo.

Nota: geralmente resulta numa menor exposição ao fármaco, muitas vezes em doses subterapêuticas, em tomas prolongadas ou múltiplas.

\section{Autorrecetor [1]}

Recetor presente numa terminação nervosa que regula, através de processos de feedback positivo ou negativo, a síntese e/ou libertação do seu próprio ligando fisiológico.

Ver também heterorrecetor.

\section{Back-up compound [2]} Ver composto de reserva.

36. Barreira hematoencefálica (BHE) [2] Camada de células endoteliais que revestem os pequenos vasos sanguíneos do cérebro.

Nota 1: estas células formam "junções apertadas" que restringem a troca livre de substâncias entre o sangue e o cérebro. Estas células são ricas em glicoproteína-P, que promove o efluxo de substratos de volta ao lado periférico da vasculatura.

Nota 2: a difusão passiva através da BHE é altamente dependente da lipofilia dos fármacos e muito poucos agentes ativos oralmente atuando no sistema nervoso central têm uma área de superfície polar maior do que $0,9 \mathrm{~nm}^{2}$.

\section{Barril beta [2]}

Estrutura secundária tridimensional de proteínas contendo uma grande folha beta que se torce e enrola para formar uma estrutura fechada na qual a primeira fita se liga à última por ligações de hidrogénio.

\section{Base de dados químicos, banco de dados químicos [2]}

Repositório eletrónico específico para armazenamento e recuperação de informação química.

Nota 1: as informações estruturais químicas são por vezes armazenadas como cadeias de caracteres ASCII, tais como as notações InChl ou SMILES.

Nota 2: estas bases de dados podem ser pesquisadas para obter informação estrutural e dados sobre moléculas específicas ou moléculas relacionadas entre si.

Nota 3: uma base de dados gratuita de estruturas químicas de pequenas moléculas orgânicas contendo informação sobre a sua atividade biológica está disponível no repositório PubChem.

\section{Biblioteca combinatória [1]}

Conjunto de compostos preparados por síntese combinatória.

\section{Biblioteca química, biblioteca de compostos, coleção de compostos [2]}

1. Coleção de amostras (ex. compostos químicos, produtos naturais, biblioteca de sobre-expressão de um micróbio) disponível para rastreio biológico.

2. Conjunto de compostos produzidos através de química combinatória ou outras abordagens e que se expandem a partir de uma estrutura-base (scaffold) ou esqueleto único.

\section{Biblioteca química virtual [2]}

Coleção de estruturas químicas construídas exclusivamente em formato eletrónico ou em papel. 
Nota: os blocos de construção necessários para tal biblioteca podem não existir, e as vias de síntese para essa biblioteca podem não ter sido investigadas. Estas coleções são usadas na conceção e avaliação de possíveis bibliotecas.

Ver rastreio virtual.

\section{Bioensaio [1]}

Procedimento para determinar a concentração, pureza e/ou atividade biológica de uma substância (ex. vitamina, hormona, fator de crescimento vegetal, antibiótico, enzima) através da determinação do seu efeito num organismo, tecido, células, enzima ou recetor, comparativamente com uma preparação de referência.

\section{Bioinformática [2]}

Disciplina abrangendo o desenvolvimento e utilização de ferramentas computacionais para armazenar, analisar e interpretar dados biológicos.

Nota: tipicamente, uma proteína ou sequência de ADN ou informação tridimensional.

\section{Bioisóstero [1] \\ Composto resultante da troca de um átomo ou grupo de átomos por outro átomo ou grupo de átomos, com características similares. 0 objetivo de uma substituição bioisostérica é o de criar um novo composto com propriedades biológicas semelhantes às do composto original. A substituição bioisostérica pode ser baseada em propriedades físico-químicas ou topológicas. \\ Ver também isóstero.}

\section{Biologia de sistemas [2]}

Integração de medidas de biologia de alta eficiência com modelos computacionais que estudam a projeção das características mecanísticas de vias metabólicas e de sinalização em características fisiológicas e fenótipos patológicos.

\begin{abstract}
46. Biologia química [2]
Aplicação da química ao estudo de eventos moleculares em sistemas biológicos, muitas vezes usando compostos como compostos-ferramenta (tool compounds).

Nota: Distingue-se da química medicinal, que se foca na conceção e otimização de compostos para alvos moleculares específicos.

Nota dos Tradutores: Compostos-ferramenta (tool compounds) são (bio)moléculas pequenas, por exemplo ARNsi ou anticorpos, que se sabe afetarem uma biomolécula (ex. um alvo terapêutico) mas cuja utilidade como fármacos é improvável, embora possam constituir pontos de partida no processo de descoberta de fármacos.
\end{abstract}

\section{Biomarcador [2]}

Indicador de sinalização de um evento ou condição num sistema ou amostra biológico(a) e fornecendo uma medida de exposição, efeito ou suscetibilidade.

\section{Biotransformação [1]}

Modificação química de substâncias por organismos vivos ou preparações enzimáticas.

\section{Boas práticas de laboratório (good laboratory practice, GLP) [2]}

Conjunto de princípios que fornece um enquadramento segundo o qual os estudos de laboratório são planeados, realizados, monitorizados, registados, reportados e arquivados.

Nota: estes estudos são realizados para gerar dados com os quais podem ser avaliados os riscos para os utilizadores, consumidores e terceiros, incluindo o meio ambiente, associados a produtos farmacêuticos (apenas estudos pré-clínicos), agroquímicos, cosméticos, aditivos alimentares, aditivos para alimentação animal e seus contaminantes, novos alimentos, biocidas, detergentes, etc. As GLP ajudam a garantir às autoridades reguladoras que os dados apresentados são um verdadeiro reflexo dos resultados obtidos durante o estudo e são, portanto, confiáveis para avaliações de risco/segurança.

\section{Boas práticas de fabrico (good manufacturing practice, GMP) [2]}

Processo de garantia de qualidade que assegura que os medicamentos são consistentemente produzidos e controlados com os padrões adequados ao uso pretendido.

Nota: os padrões de qualidade são os exigidos para autorização de comercialização ou pela especificação do produto. As GMP estão relacionadas tanto com a produção como com o controlo de qualidade.

\section{Bomba de efluxo [2]}

Proteína transportadora localizada na membrana celular e que utiliza o transporte ativo para mover um composto do ambiente interno para o externo.

Ver também ATP binding cassete, glicoproteína-P.

As entradas 52. a 305. podem ser consultadas em material suplementar, glossário de termos completo, disponível em spq. pt/files/files/BSPQ/N158-artigo-22.pdf.

\section{Validação do alvo [2]}

Processo pelo qual uma proteína, o ARN ou o ADN são implicados numa via biológica considerada relevante para uma doença ou uma patologia adversa.

Nota: normalmente, a validação envolverá a localização do alvo molecular em células, órgãos ou tecidos relevantes, evidências da sua regulação ascendente/ ativação na patologia, e a capacidade para atenuar as respostas adversas provocadas por agentes conhecidos por interferir com o alvo. 


\section{Validação do composto-líder ou protótipo [2]}

Processo pelo qual um composto-líder ou protótipo é autenticado pela confirmação das suas propriedades farmacológicas previstas.

Nota: geralmente, um conjunto de compostos estruturalmente semelhantes mostrando uma relação estrutura-atividade percetível reforçará o processo de validação.

\section{Virtual screening [2] Ver rastreio virtual.}

309. Volume de distribuição $\left(V_{\mathrm{d}}\right)[2]$

Volume aparente (hipotético) de fluido necessário para conter a quantidade total de uma substância no corpo na mesma concentração em que está presente no plasma assumindo que o equilíbrio foi alcançado.

\section{Xenobiótico [1]}

Composto estranho a um organismo (xenos [grego]= estranho).

\section{Referências}

[1] C. G. Wermuth, C. R. Ganellin, P. Lindberg, L. A. Mitscher, Pure Appl. Chem. 1998 70, 1129-1143. DOI: 10.1351/pac199870051129.

[2] D. R. Buckle, P. W. Erhardt, C. R. Ganellin, T. Kobayashi, T. J. Perun, J. Proudfoot, J. Senn-Bilfinger, Pure Appl. Chem. 2013, 85, 1725-1758. DOI: 10.1351/PACREC-12-11-23.

\section{Jorge Salvador}

É Professor Catedrático da Faculdade de Farmácia da Universidade de Coimbra, líder do grupo de Medicinal Chemistry \& Drug Discovery no Centro de Neurociências e Biologia Celular da Universidade de Coimbra (CNC-UC), membro do consórcio CIBB (Center for Innovative Biomedicine and Biotechnology). salvador@ci.uc.pt ORCID.org/0000-0003-0779-6083

$>$

\section{Matilde Marques}

É Professora Catedrática do Instituto Superior Técnico da Universidade de
Lisboa. Desenvolve a sua investigação no Centro de Química Estrutural, onde coordena a linha temática MEDLife - Medicinal and Biological Chemistry for Health. Foi Presidente da Divisão de Química Terapêutica da SPQ entre 2016 e 2019. matilde.marques@tecnico.ulisboa.pt ORCID.org/0000-0002-7526-4962

\section{Madalena Pinto}

É Professora Catedrática Jubilada da Faculdade de Farmácia da Universidade do Porto. Desenvolve o seu trabalho de

\section{Agradecimentos}

Os autores agradecem aos representantes da IUPAC e SPQ pelo valioso apoio, comentários ou sugestões: Amélia Rauter (Universidade de Lisboa), Artur Silvaiii (Universidade de Aveiro), Augusto Tomé (Universidade de Aveiro) e Paula Gomesiv (Universidade do Porto).

\section{Notas}

' Tradução adaptada das recomendações da International Union of Pure and Applied Chemistry (IUPAC) 1998, 2013 [1,2]

ii a) Quando os termos se encontram definidos no glossário de 1998 e no de 2013 optou-se pela terminologia do glossário de 2013; b) As palavras a negrito correspondem a entradas no g'lossário; c) Foram mantidas entradas em língua inglesa no glossário nos casos em que os termos são frequentemente usados nesta língua.

iii Presidente da Sociedade Portuguesa de Química (SPQ) no período em que este trabalho foi preparado.

ivepresentante da IUPAC (Division: VII Chemistry \& Human Health) no período em que este trabalho foi preparado. investigação e é Líder do Grupo de Produtos Naturais e Química Medicinal no Centro Interdisciplinar de Investigação Marinha e Ambiental (CIIMAR)

madalena@ff.up.pt

ORCID.org/0000-0002-4676-1409

\section{$>$}

\section{* Maria Emília Sousa}

É Professora Associada com Agregação da Faculdade de Farmácia da Universidade do Porto. Desenvolve o seu trabalho de investigação no Centro Interdisciplinar de Investigação Marinha e Ambiental. É Presidente da Divisão de
Química Terapêutica da SPQ desde 2020 esousa@ff.up.pt ORCID.org/ 0000-0002-5397-4672

\section{Rui Moreira}

É Professor Catedrático da Faculdade de Farmácia da Universidade de Lisboa, líder do grupo de Química Medicinal no Instituto de Investigação do Medicamento (iMed.ULisboa), e é Presidente Efetivo da European Federation for Medicinal Chemistry (EFMC). rmoreira@ff.ulisboa.pt orcid.org/0000-0003-0727-9852 